\title{
A Proposed Ascent Abort Flight Test for the Max Launch Abort System
}

\author{
Paul. V. Tartabini ${ }^{1}$ Michael G. Gilbert ${ }^{2}$ and Brett R. Starr ${ }^{3}$ \\ NASA Langley Research Center, Hampton, Virginia 23681
}

\begin{abstract}
The NASA Engineering and Safety Center initiated the Max Launch Abort System (MLAS) Project to investigate alternate crew escape system concepts that eliminate the conventional launch escape tower by integrating the escape system into an aerodynamic fairing that fully encapsulates the crew capsule and smoothly integrates with the launch vehicle. This paper proposes an ascent abort flight test for an all-propulsive towerless escape system concept that is actively controlled and sized to accommodate the Orion Crew Module. The goal of the flight test is to demonstrate a high dynamic pressure escape and to characterize jet interaction effects during operation of the attitude control thrusters at transonic and supersonic conditions. The flight-test vehicle is delivered to the required test conditions by a booster configuration selected to meet cost, manufacturability, and operability objectives. Data return is augmented through judicious design of the boost trajectory, which is optimized to obtain data at a range of relevant points, rather than just a single flight condition. Secondary flight objectives are included after the escape to obtain aerodynamic damping data for the crew module and to perform a high-altitude contingency deployment of the drogue parachutes. Both 3- and 6-degree-of-freedom trajectory simulation results are presented that establish concept feasibility, and a Monte Carlo uncertainty assessment is performed to provide confidence that test objectives can be met.
\end{abstract}

\section{Nomenclature}

ConOps $\quad=$ Concept of Operations

c.g. $\quad=$ center of gravity

Q-Alpha-Total $=$ Product of dynamic pressure and total angle of attack

\section{Introduction}

A S NASA pursues both commercial and government options for transporting humans to destinations in low earth orbit and beyond, crew safety remains a primary objective. An important element of this goal is NASA's requirement that all human-rated space transportation systems provide the capability for crew escape while on the pad and at any time from launch to orbit insertion.

To meet this goal, the Orion Multi-Purpose Crew Vehicle (MPCV), which is currently under development, has adopted a tower-based escape system configuration that builds on the heritage of the Mercury and Apollo systems. ${ }^{1-2}$ The Orion Launch Abort System (LAS) improves upon the earlier tower-based designs by utilizing an Attitude Control Motor for active control to guide and stabilize the LAS during an abort and to perform a 180-degree reorientation maneuver prior to parachute deployment. The use of active control enables reductions in the length and weight (due to ballast elimination) of the escape system, below that of purely passive tower-based heritage systems. ${ }^{3}$

Further benefits can be attained by eliminating the single-motor escape tower altogether. The NASA Engineering and Safety Center (NESC) initiated the Max Launch Abort System (MLAS) Project in 2007 that investigated crew escape system vehicle concepts that did not make use of the conventional tower-configuration approach. ${ }^{4}$ The MLAS escape vehicle proposed in the NESC study ${ }^{5}$ eliminates the escape tower by fully encapsulating the escape system within an aerodynamic fairing that smoothly joins with the capsule. By removing the tower, the integration of the MLAS escape system and capsule with the launch vehicle is greatly improved, resulting in reductions in aerodynamic

\footnotetext{
${ }^{1}$ Aerospace Engineer, Vehicle Analysis Branch, 1 North Dryden Street, Mail Stop 451, Member.

${ }^{2}$ Principal Engineer, NASA Engineering and Safety Center, 11 Langley Blvd, Mail Stop 116, Associate Fellow.

${ }^{3}$ Aerospace Engineer, NASA Langley Vehicle Analysis Branch, 1 North Dryden Street, Mail Stop 451, Member.
}

1 
drag and structural bending moments during ascent, and a decrease in the amount of induced aero-acoustic loads. These improvements in the integrated launch vehicle can lead to significant structural weight savings and an increased payload capacity. The MLAS escape vehicle concept and the Orion LAS are compared in Fig. 1.

Because of the emphasis that NASA has placed on crew safety, extensive flight testing will likely be required for any escape vehicle before a crew is committed. Indeed, successful flight demonstrations were required during Projects Mercury and Apollo, and the Orion program has already performed a pad-abort test of the LAS and has plans to conduct an ascent abort test in the future. In the case of Orion, even though the LAS is a heritage design, it is still regarded by NASA as different enough from its predecessors that flight testing at both pad and ascent abort conditions is considered necessary for validating its capabilities.

Similar flight tests would certainly be required for the towerless MLAS escape system, which is substantially different from the LAS or previous NASA heritage escape systems. Chief among these differences is the use of multiple aft-mounted escape motors to accelerate away from a failing launch vehicle and multiple liquid attitude control system (ACS) thrusters that are continuously turned on and off to generate control moments. These propulsion systems will have to operate across the Mach regime in complex, interacting flow conditions. Performance will be subject to large uncertainties due to near-field separation aerodynamics, power-on plume effects, and aeropropulsive jet interactions. Such phenomena cannot be adequately tested in wind tunnels or modeled computationally without data collected from flight tests in realistic dynamic environments.

This paper describes a proposed ascent-abort flight test for the MLAS escape system ${ }^{4}$. The primary objectives of the test are to demonstrate escape capability at a high dynamic pressure stress condition and to obtain forward flight aerodynamic and ACS jet interaction data in the transonic and low supersonic flight regimes. In addition, steps taken to maximize the value of the proposed test are discussed. For instance, through careful tailoring of the boost and escape vehicle trajectories, data can be obtained at a range of relevant points rather than just a single flight condition. In addition, the escape sequence lends itself to the addition of secondary test objectives that can benefit the Orion Program such as collecting aerodynamic damping data for the crew module (CM) and performing a high altitude parachute deployment to help quantify Capsule Parachute Assembly System (CPAS) margins.

\section{MLAS Description}

The MLAS escape vehicle discussed in this paper is based on the all-propulsive concept described in Ref. 5. A drawing of the vehicle is shown in Fig. 2. Three different propulsion systems are integrated within an aerodynamically shaped fairing that encapsulates the Orion CM. The fairing utilizes a modified Sears-Haack shape ${ }^{6}$ that acts as an aerodynamically clean, low-drag front end to the integrated launch vehicle. It is attached to the CM at six points near the middle of the backshell cone and is designed to carry and transmit the structural loads due to aerodynamic and propulsive forces encountered during nominal ascent and abort.

Figure 3 shows the layout and dimensions of the MLAS vehicle. Six solid rocket escape motors are positioned circumferentially around the fairing at 60-degree intervals and are canted outward 30 degrees. Thrust levels are tailored to meet escape requirements while limiting crew exposure to high accelerations. Each escape motor is equipped with thrust vector control (TVC), and nozzles can be deflected up to 15 degrees in any direction outward of the fairing at a rate of 200 degrees/second. The nozzles are not permitted to deflect inward to minimize plume impingement.

\footnotetext{
${ }^{4}$ An aerodynamically stabilized, uncontrolled variant of MLAS was pad-abort tested in July 2009, see for example Ref. 12.
} 


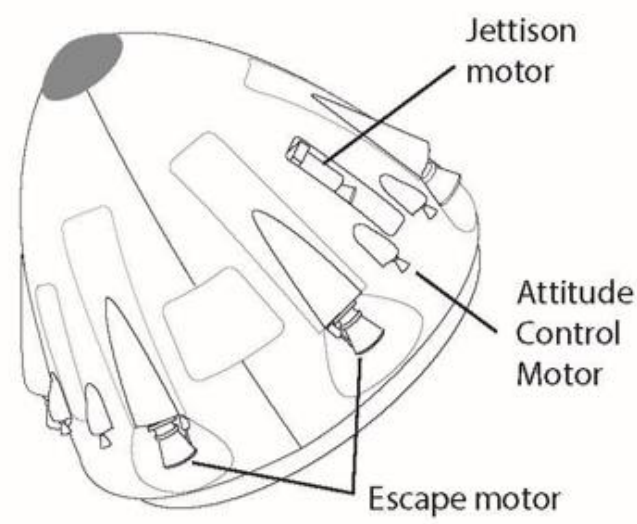

Figure 2. MLAS Escape Vehicle Concept.

Eight hypergolic liquid ACS engines provide control during coast and reorientation phases. The ACS thrusters are activated during escape motor tailoff and operate for the remainder of the escape trajectory to stabilize the vehicle and perform a 180-degree reorientation to a heat shield forward attitude. The placement of the ACS thrusters is shown in Fig. 3. Each thruster is canted outward 15 degrees and has an additional slight tangential cant angle to provide a small amount of roll control. The thrusters can be turned on and off in various combinations (with a minimum on/off time of 50 $\mathrm{ms}$ ) to produce control moments and are cycled on and off numerous times in a typical escape trajectory.

Normally the CM has a small radial center of gravity (c.g.) offset during launch to enable a lifting trajectory during nominal re-entry or in case of a high-altitude abort re-entry. To increase the escape system ACS control margins, the escape hardware has $1,000 \mathrm{lbm}$ of ballast added on one side of the escape fairing to compensate for the CM c.g. offset. Trajectory analysis indicates that it may be possible to achieve acceptable control performance with less ballast, but additional study is needed.

Three solid rocket jettison motors are used to remove the escape system fairing from the $\mathrm{CM}$ at the end of an abort when the CM has been maneuvered to a safe distance from the launch vehicle. These motors are also used to remove the unused escape system in a nominal ascent after it is no longer needed. Space Shuttle booster separation motors, originally utilized for the solid rocket booster separation, were used as jettison motors. The jettison motors had to be canted outwards at least 45 degrees to avoid plume impingement; however, each individual motor's cant angle was different to produce a resultant thrust moment that provided adequate separation performance.

The MLAS was sized to provide acceptable escape performance assuming a representative CM capsule weighing $\sim 21,400 \mathrm{lbm}$. To accommodate this CM weight, the design of the escape system (fairing, motors, and propellant) weighs approximately $15,000 \mathrm{lbm}$.

\section{Ascent Abort Flight Test}

An ascent abort flight-test demonstration is proposed to validate the functionality of the MLAS towerless launch escape vehicle concept. The primary test objective is to demonstrate escape from the booster with adequate clearance at a high dynamic pressure stress condition. For this study, the abort initiation target condition is a dynamic pressure of $760 \mathrm{psf}$ and a Mach number between 1.4 and 2.0. This target provides an escape environment that is similar to the peak dynamic pressure condition of NASA's Space Launch System trajectory with dispersions above its nominal value. 
The specific test objectives defined to validate system functionality include executing an escape that meets the Orion LAS ascent abort requirement of achieving at least 175 feet of separation from the booster within 3 seconds of escape initiation, performing a controlled coast and reorientation of the escape vehicle using ACS thrusters, and successfully jettisoning the escape fairing from the $\mathrm{CM}$ at the end of the escape trajectory. Along with demonstrating ACS operation, an additional objective is to collect aerodynamic and jet interaction data during forward coasting flight in the subsonic, transonic, and supersonic flow regimes.

To increase the potential benefit of the flight test, two secondary test objectives were added. First, an additional CM free-flight segment is included after the escape to obtain aerodynamic data. Specifically, after escape fairing jettison, an uncontrolled CM coast is performed with induced pitch oscillations to gather aerodynamic damping data while staying within parachute deployment limits. Second, the CPAS parachutes are deployed at the end of the CM coast segment at an altitude of 35,000 feet, to quantify performance margins at the upper bound of the CPAS operating envelope. All of the flight-test objectives are summarized in Table 1.

Table 1. Test Objectives for Proposed MLAS Ascent Abort Flight Test

\begin{tabular}{|c|c|}
\hline Test Objective & Priority \\
\hline $\begin{array}{l}\text { - Demonstrate escape from the booster with adequate clearance } \\
\text { at a high dynamic pressure stress condition. } \\
\text { - Achieve at least } 175 \text { feet of separation distance from the } \\
\text { booster within } 3 \text { seconds after escape initiation. } \\
\text { - Perform a controlled coast and reorientation of the escape } \\
\text { vehicle using ACS thrusters, and demonstrate ACS operation } \\
\text { in subsonic, transonic, and supersonic flow regimes. } \\
\text { - Demonstrate jettison of the escape fairing from the CM at the } \\
\text { end of the escape trajectory. }\end{array}$ & Primary \\
\hline $\begin{array}{l}\text { Perform an uncontrolled CM coast with sufficient pitch } \\
\text { oscillations to gather aerodynamic damping data while staying } \\
\text { within parachute deployment limits. } \\
\text { - Deploy the CPAS parachutes at an altitude of } 35,000 \text { feet and } \\
\text { gather data necessary to quantify performance margins. }\end{array}$ & Secondary \\
\hline
\end{tabular}

The Concept of Operations (ConOps) for the proposed ascent abort flight test is shown in Fig. 4. The MLAS flighttest vehicle (FTV), which includes the escape fairing and CM test capsule, is delivered to the escape initiation condition by a custom built booster (described in the next section). As the booster motor thrust tails off, the FTV's six escape motors ignite, and a controlled pitch maneuver is performed using TVC to induce a lateral separation and maximize the separation distance from the booster's flight path. As the thrust of the solid rocket escape motors begin to tail off prior to burnout, the liquid ACS thrusters become operational and act jointly with the escape motor TVC to stabilize the vehicle and counteract thrust dispersions. After the escape motors fully burn out, the control thrusters continue to maintain a stable coast until the vehicle is close to apogee, when a reorientation maneuver is performed to properly position the CM for parachute deployment. After reorientation, the ACS thrusters damp out residual body rates until a stable heat shield forward attitude is attained. The FTV continues to hold this attitude and coast under ACS control until the fairing jettison altitude is reached, at which time the ACS thrusters are shut off and the jettison motors ignite to separate the escape fairing from the CM, placing it on a trajectory that avoids interference with the $\mathrm{CM}$. After fairing jettison, the CM performs an uncontrolled coast to acquire aerodynamic damping data until reaching an altitude of 35,000 feet when the CPAS parachute deployment sequence is initiated.

The main differences between the flight-test ConOps described above and the baseline ConOps for an MLAS midaltitude escape (described in Ref. 5) occur after reorientation. In the baseline ConOps, the escape fairing is jettisoned much sooner and the CM descends under its own reaction control system (RCS) control until parachutes are deployed; however, in the proposed flight test the CM will not have RCS or its own control system (to reduce costs), and the FTV coast segment must be extended so that the ACS thrusters can maintain stability. When the escape fairing is jettisoned, the CM performs an uncontrolled free-flight coast and acquires aerodynamic damping data. The major phases in the ascent abort flight test, including the CM free-flight phase, are described in more detail below. 


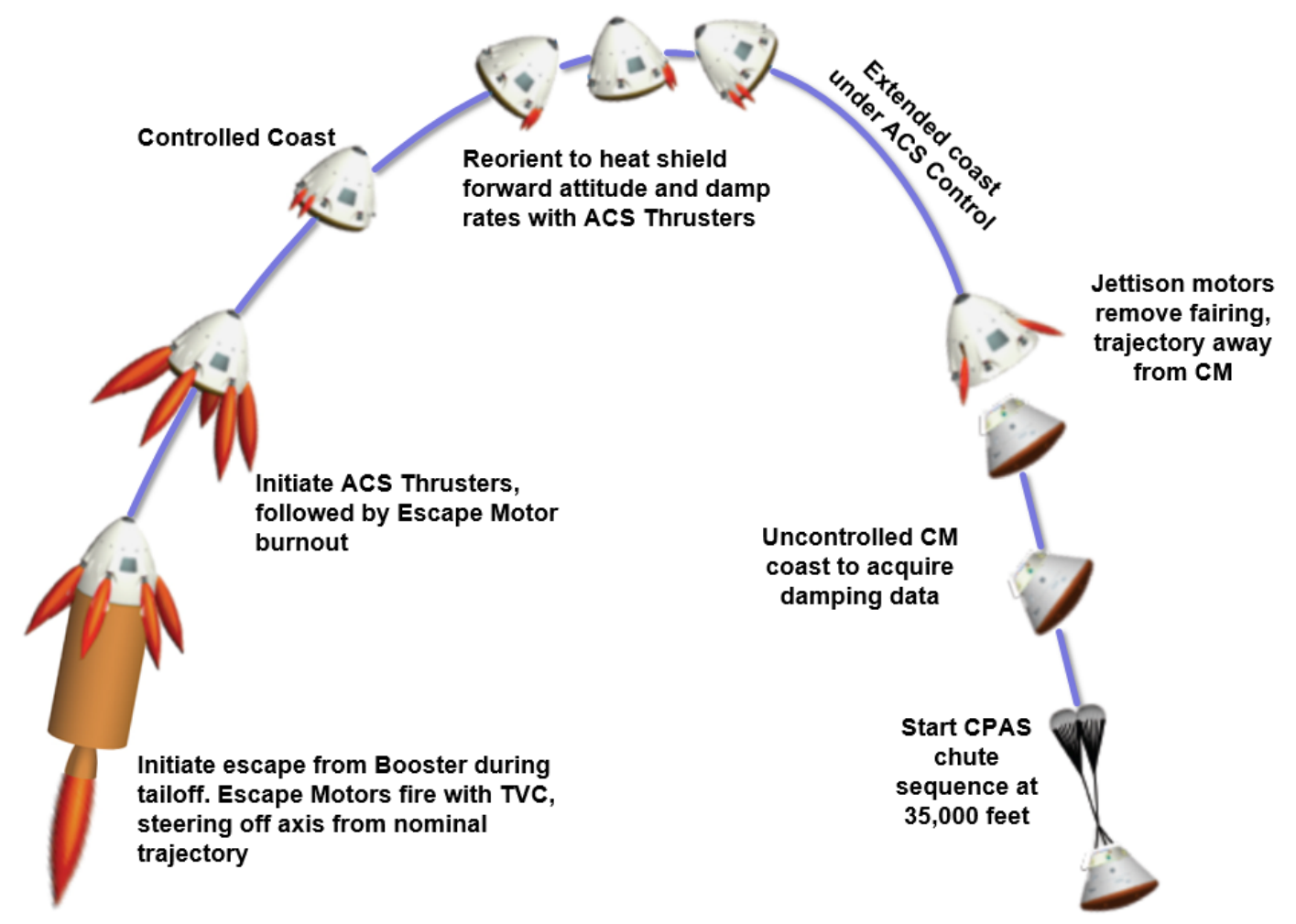

Figure 4. Concept of Operations for Ascent Abort Flight Test.

\section{A. Booster Segment}

The MLAS FTV will be delivered to the desired escape test initiation condition with a custom-built booster. Studies of booster configurations with one or more solid rocket motors were conducted to identify a booster that would provide low supersonic, transonic, and subsonic flight conditions during the escape trajectory. The booster configurations that were evaluated employed excess, current production, and developmental motors used in civilian and military applications. The motors had a wide range of total impulse, ranging from 0.3 to $30 \mathrm{Mlb}$-s. The larger impulse motors ( $>10 \mathrm{Mlb}$-s) were evaluated in a booster configured for a monolithic core motor. The small diameter, lower impulse (<6 Mlb-s) motors were evaluated in a booster configured for multiple core motors. The intermediate impulse motors between those ranges were evaluated in a booster configured for a single core motor with strap-ons.

All booster configurations consisted of a cylindrical aerodynamic skirt that enclosed the motors and the mounting structure that attached the motors to the skirt and FTV capsule. The skirt diameter was equal to the base diameter of the FTV capsule fairing and was attached to the FTV fairing. The aft end of the skirt was attached to the motors through mounting structure. Mounting structure was also used to transmit the motor thrust force into the CM capsule's hard points that are normally used to mount it to the service module. The skirt length and motor mounting structure were varied to accommodate the various motor configurations. Representative sketches of the booster configurations are shown in Fig. 5.

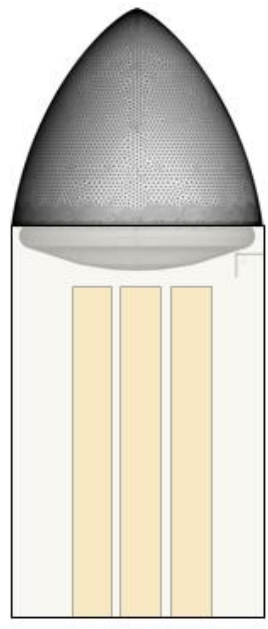

Multiple Cores

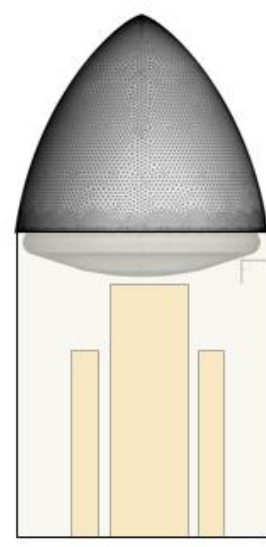

Core w/Strap-ons

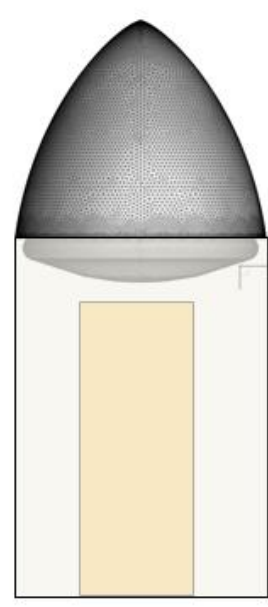

Monolithic Core

Figure 5. Representative sketches of booster configurations considered for ascent abort flight test. 
Developing a custom booster and tailoring the boost trajectory provided an effective way to affect the escape test segment of the trajectory to better achieve flight-test objectives and maximize data return. Three-degree-of-freedom trajectory analysis that modeled the boost trajectory and included the FTV test segment was performed for each candidate booster using the Program to Optimize Simulated Trajectories II (POST2). ${ }^{7}$ The booster weight and the trajectory pitch profile were optimized to place the FTV within the desired Mach-dynamic pressure flight-test initiation ranges and maximize the time at which the ACS thrusters operated at transonic and low supersonic speeds in a forward flight orientation (before reorientation).

The results of these analyses showed that all configurations (multiple cores, core with strap-on, and monolithic core) could meet the target state with an appropriate arrangement of the available motors. Thus, cost, manufacturability, and operability became the key considerations in selecting the booster configuration and its motor. A monolithic core configuration was chosen because it was less complex to manufacture, integrate, and operate. A motor in the 250,000-lbf thrust class with a total impulse of $\sim 17 \mathrm{Mlb}$-s was selected due to its performance and cost benefits. The motor performance was sufficient to meet the targeted condition for a booster structure constructed of either aluminum or steel. At the time of this study, a developmental motor with these thrust and impulse characteristics was available at a reduced cost because its use would have flight-test benefits for the manufacturer.

Two Mach versus dynamic pressure profiles for the entire flighttest trajectory (boost and escape) are shown in Fig. 6 for the selected monolithic core motor: one assuming a booster structure of steel and the other of aluminum. A nominal abort initiation Mach number of 1.6 is attained with steel construction and 1.9 for the lighter aluminum construction (both are within the $1.4 \leq$ Mach $\leq 2.0$ target range). Either option provides adequate coverage of transonic/low supersonic ACS operation during forward coasting flight.

Based on this analysis, the baseline approach was to use steel for the booster structure. In the event of weight growth during design maturation, the structure could be made with a combination of steel and aluminum to keep the performance the same. Moreover, the option to make

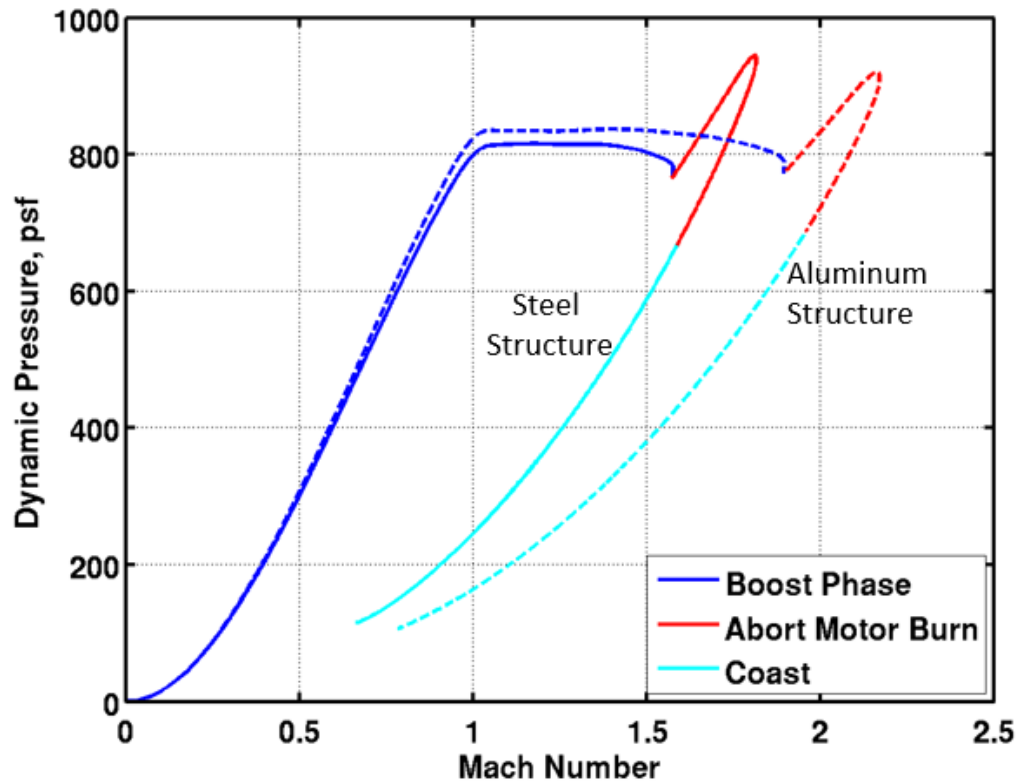

Figure 6. Comparison of combined boost and escape trajectories for the chosen booster configuration assuming a booster structure consisting of steel (solid line) and aluminum (dashed line).

the structure entirely out of aluminum remained as a way to increase the abort initiation Mach and thus expand the time of ACS operation within the transonic/low supersonic range.

\section{B. Escape Flight-Test Segment}

To assess the feasibility and controllability of the FTV, a 6-degree-of-freedom simulation of the entire escape trajectory sequence was developed using POST2. The Mach versus dynamic pressure profile of the entire trajectory including boost and parachute deployment is shown in Fig. 7 and time histories of altitude, Mach, dynamic pressure and angle-of-attack (alpha) are shown in Fig. 8.

The escape test is initiated during the tailoff of the booster motor at an altitude of $\sim 41,000 \mathrm{ft}$, Mach number of 1.6, and dynamic pressure of $760 \mathrm{psf}$. The FTV separates and rapidly accelerates during the initial part of the escape motor burn and then begins to decelerate as the motors tailoff. The ACS thrusters are activated during tailoff, 3 seconds after escape-motor ignition, near Mach 1.8. Escape-motor burnout occurs 7 seconds after ignition at Mach 1.45, at which point forward coasting flight under full ACS control commences. From this point, until the reorientation maneuver is performed at Mach 0.7, aerodynamic and jet interaction data are collected. 


\begin{tabular}{|ll|}
\hline 1. & Abort Initiation \\
2. & Begin ACS Control \\
3. & Start Reorientation \\
4. & Fairing Jettison \\
5. & Drogue Deploy \\
6. & Main Deploy \\
\hline
\end{tabular}

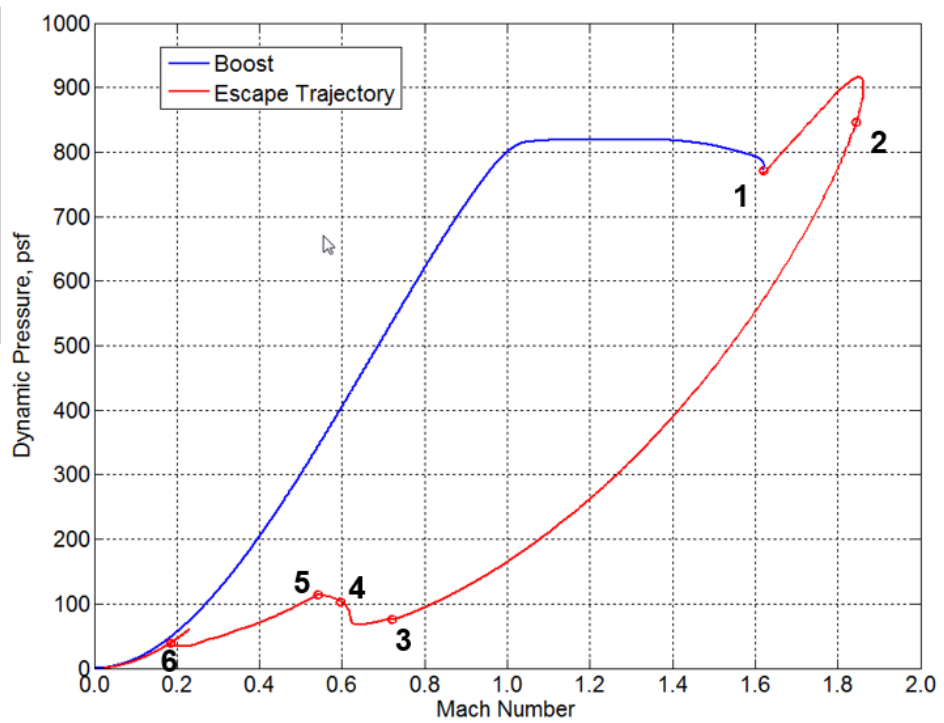

Figure 7. Flight-test trajectory, dynamic pressure versus Mach.
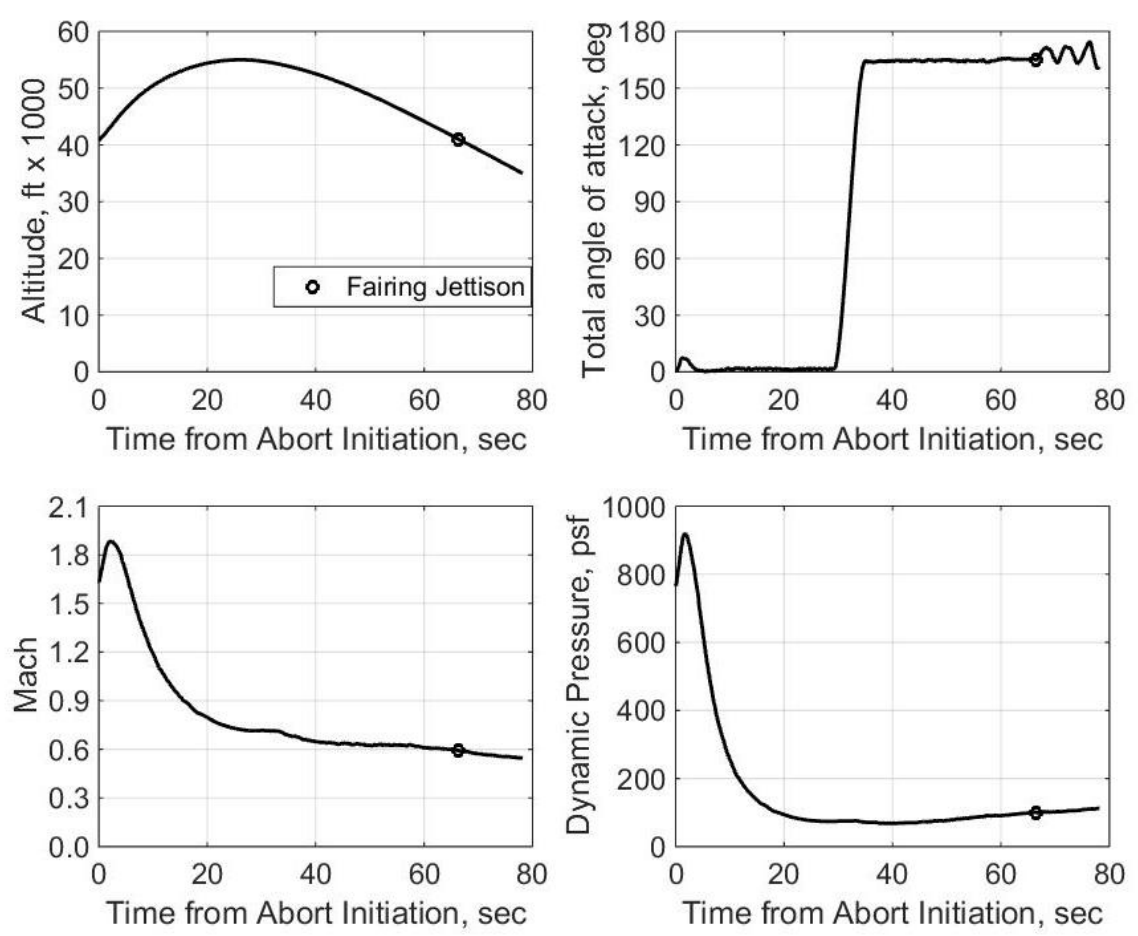

Figure 8. Nominal trajectory results for the escape segment of the flight-test trajectory.

At the start of the escape-motor burn, a pitch maneuver is performed using TVC to increase the lateral distance from the booster trajectory and ensure that the 175 -foot separation distance requirement is met. During this maneuver, the FTV reaches a peak alpha of 7 degrees. Throughout the escape trajectory, Q-Alpha-Total (the product of dynamic pressure and total alpha) is kept below 10,000 psf-deg to stay within vehicle structural load limits. When the ACS thrusters are activated, the FTV transitions to 0-degree alpha and holds this attitude for the duration of the prereorientation coast. Roughly 29 seconds after escape-motor ignition, reorientation occurs near the apogee altitude of 55,000 feet when dynamic pressure is nearly minimized, and the ACS thrusters are used to reorient the FTV from a nose-forward to a heat shield forward attitude. After reorientation is complete, the FTV targets and holds an attitude that is slightly offset from the CM's heat shield forward trim alpha. Escape fairing jettison occurs at 41,000 feet, at which time the CM begins its uncontrolled free-flight segment. Because the CM is initially offset from its trim alpha,

American Institute of Aeronautics and Astronautics 
it experiences pitch oscillations, which are necessary for obtaining CM aerodynamic damping data. The parachute deploy sequence beings 12 seconds later at an altitude of 35,000 feet, when the drogue parachutes are deployed. Main parachute deployment follows shortly thereafter, and the CM descends to a water landing roughly 12 nautical miles away from the launch pad.

The net pitch and yaw control moments generated by the ACS thruster are shown in Fig. 9. The thrusters must be cycled on and off numerous times to maintain control throughout the trajectory. For the nominal case, the number of on/off cycles varies among the thrusters with the least active cycling on and off $\sim 40$ times, and the most active $\sim 135$ times. The control moment results in Fig. 9 show that there are frequent ACS cycles prior to reorientation when jet interaction data will be obtained. Despite the high activity of the ACS thrusters over the entire trajectory, just over half of the available propellant is used in the nominal case.
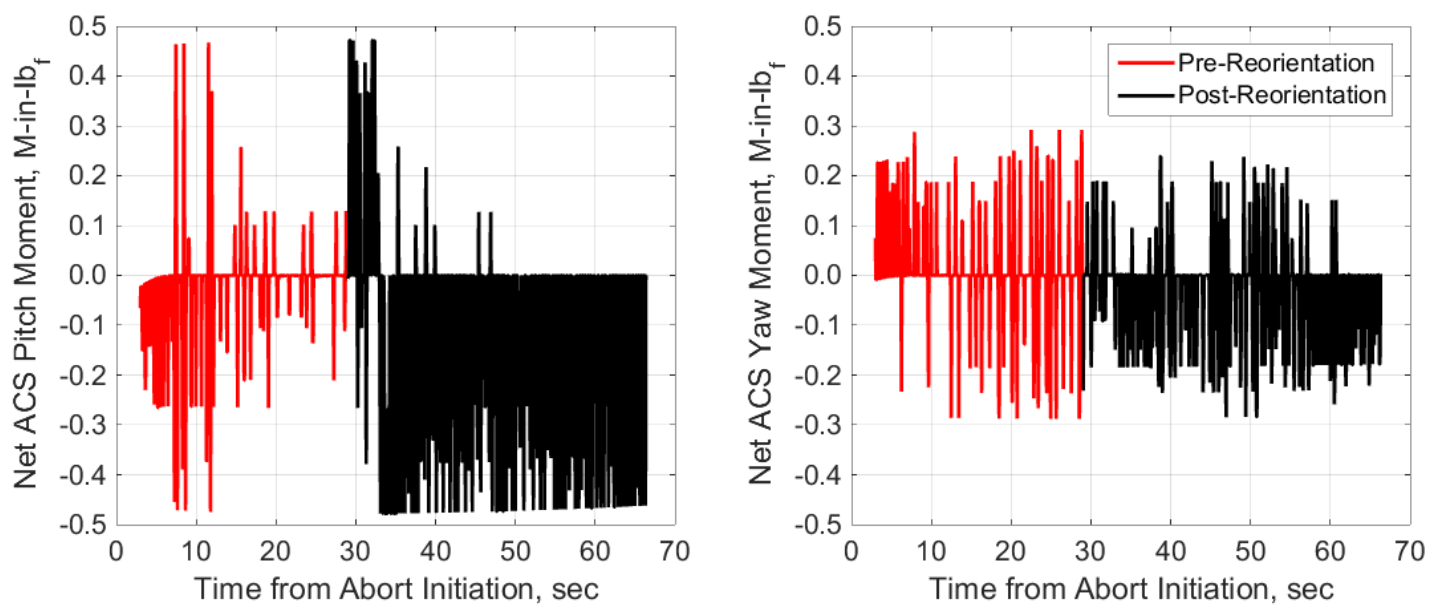

Figure 9. Net pitch and yaw ACS control moments for the nominal escape test trajectory.

To assess the separation distance requirement, trajectories were run that tracked the booster after escape initiation. Due to a lack of aerodynamic data for the booster configuration without the FTV attached, both low and high booster drag models were assumed to estimate the separation distance. The low-drag model was conservative and assumed that there was no increase in booster drag after separation of the FTV. The separation distance results (Fig. 10) show that the separation distance is much higher than the 175-foot requirement 3 seconds after abort initiation, suggesting that there is adequate separation margin in the design. The uncertainty associated with escape vehicle separation, especially due to aerodynamic interference and potential suction drag effects, underscores the importance of doing an ascent abort flight test.

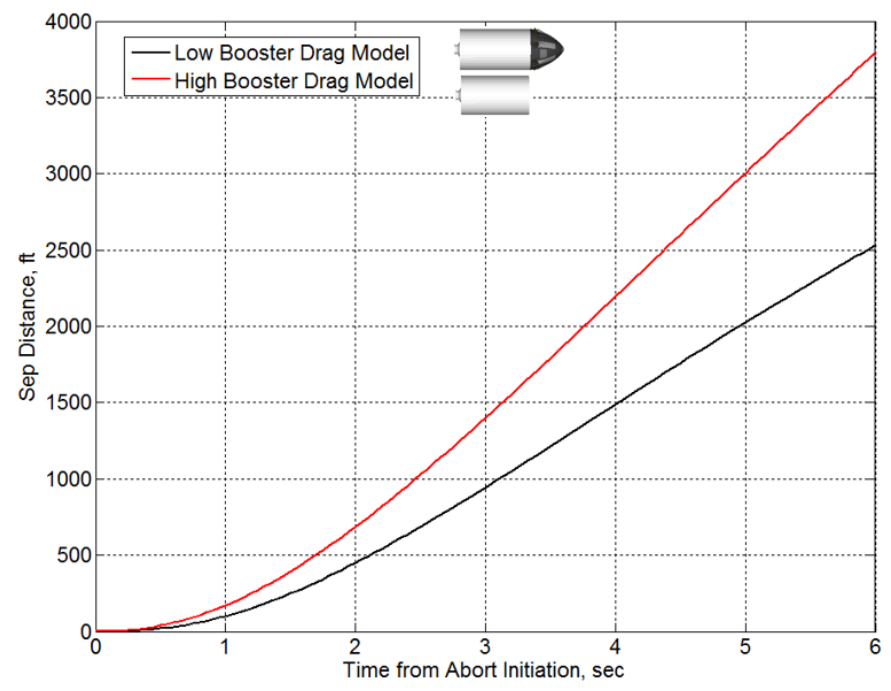

Figure 10. Comparison of predicted separation distance for low and high booster drag models.

\section{Crew Module Coast Segment}

To increase the potential benefit of the flight test, two secondary objectives were added to obtain CM aerodynamic damping data and perform a parachute deployment at the upper altitude bound of the CPAS operating envelope. Both of these flight segments (CM free-flight and parachute deployment) were already part of the MLAS ascent abort ConOps, but were modified in the flight test as described below to increase their potential data value.

During nominal operation, the CM must tolerate periods of uncontrolled free flight that occur in an abort (between escape fairing jettison and drogue deploy) and in an entry (while drogue parachutes are deployed and then again between drogue release and main parachute inflation). These free-flight segments occur when the CM is dynamically 
unstable (heat shield forward attitude at subsonic conditions) and may cause the CM to tumble during uncontrolled free flight. ${ }^{8}$

To characterize this dynamic instability, the MPCV Program has conducted numerous ground-based tests using a variety of dynamic test techniques as described in Ref. 8. However, accurate prediction of aerodynamic damping coefficients require high Reynolds numbers and close adherence to a range of similitude parameters, both of which are hard to achieve in ground-based tests. Thus, obtaining free-flight damping data to validate and anchor existing test points is of great interest.

In addition to obtaining CM damping data, another objective was to deploy the CPAS drogue parachutes at the desired test conditions for a 35,000-foot contingency drogue deployment. Drop tests have been done above the nominal 25,000-foot deployment altitude, but not with deployment at 35,000 feet, which is at the upper edge of the drogue deployment envelope. This addition to the MLAS flight test would provide valuable data that could anchor Orion models at the upper deployment altitude.

To meet these secondary test objectives, the escape sequence was modified in several ways compared to the baseline sequence defined in the MLAS ascent abort ConOps from Ref. 5. First, after reorientation, the FTV targets an alpha that is offset from the trim location of the CM (as opposed to targeting the trim alpha itself, as is done in a typical abort). The trim offset is necessary for inducing the desired motion during the CM free flight. Second, the postreorientation coast time of the FTV is extended beyond that of a nominal escape because, for cost and simplicity reasons, the CM test capsule will not have a control system in the flight test. This differs from a nominal escape where the fairing is jettisoned as soon as residual rates have been removed and the CM descends under its own RCS control. Finally, the duration of uncontrolled CM free flight prior to deployment of the drogue parachutes is expanded in the flight test so that aerodynamic damping data can be obtained. In a typical abort this free-flight segment is much shorter, lasting only long enough to ensure that the jettisoned escape fairing has cleared the vicinity of the CM.

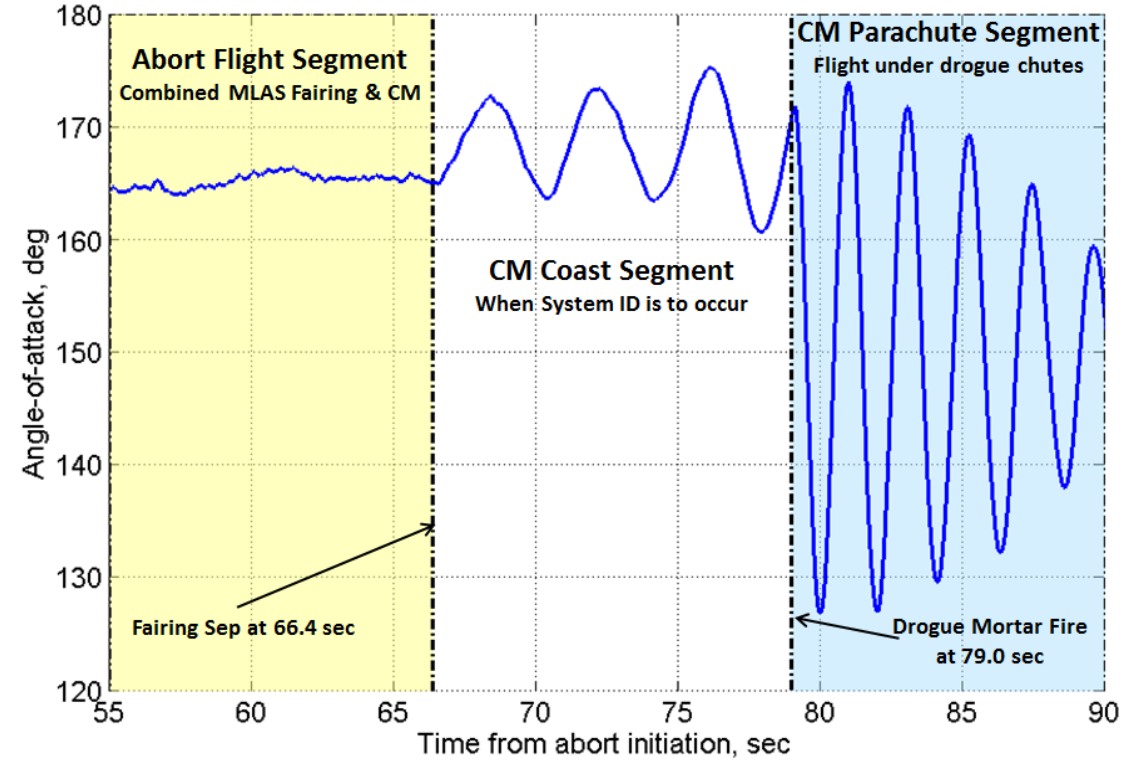

Figure 11. Timeline of CM free flight-test segment.

The timeline for the $\mathrm{CM}$ free-flight test segment is shown in Fig. 11. During the abort flight segment the ACS holds the FTV at the desired alpha (offset several degrees from the CM trim alpha). Once the escape fairing is separated, the CM coasts uncontrolled and oscillates around its trim point. During this period of free response, rate and acceleration data are recorded by the onboard inertial navigation system (INS) and can be used to estimate the static and dynamic stability derivatives of the $\mathrm{CM}$ using system identification techniques. ${ }^{9}$ The coast segment ends at drogue deployment and is timed such that the drogues are fully deployed at an altitude of 35,000 feet. If oscillations grow too large during the CM coast, the parachute deployment sequence can be initiated early to prevent violation of drogue deploy constraints.

To define the characteristics and duration of the CM coast segment, system identification analyses were performed using simulation data. The amount and quality of data obtained depends on the duration of the coast and the size of the pitch oscillations. To accurately estimate aerodynamic coefficients, it was determined that at least three pitch oscillations would be required. In addition, the range of data that could be collected within the alpha/pitch rate subspace depends on the size of the oscillations, which is affected by the amount of the trim offset (see Fig. 12). To best characterize the aerodynamic damping of the CM, large pitch oscillations are desired; however, if the amplitude is too high or the coast period is too long, then the CM could exceed the 130-degree drogue deployment constraint on total alpha and begin to tumble. 


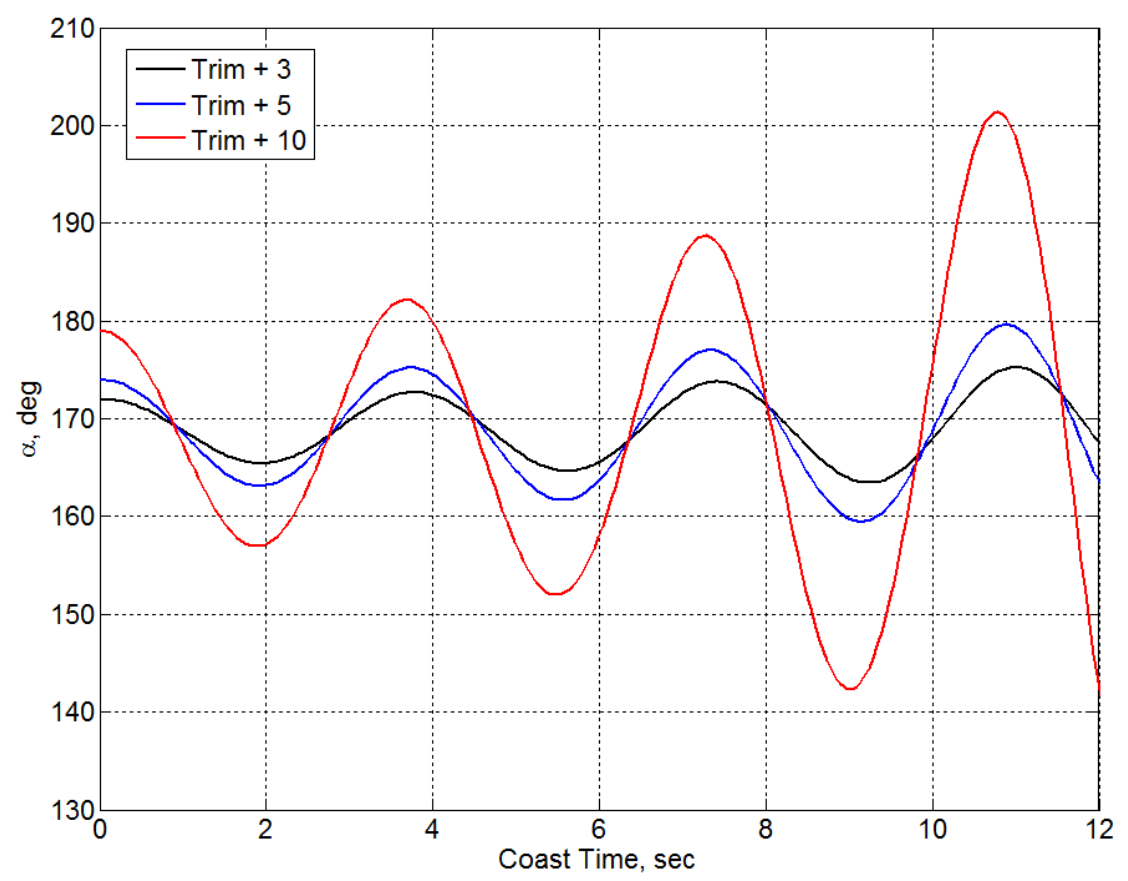

Figure 12. Comparison of CM response to different trim offsets.

To balance these competing effects, it was decided to jettison the fairing at an altitude of 41,000 feet with a 3-degree trim offset. This sequence provides for three CM pitch oscillations over a coast period of 12 seconds and allows for a drogue deployment at the target altitude of 35,000 feet. Targeting the trim offset alpha precisely will be difficult due to wind dispersions as well as uncertainty in the trim angle itself, due to aerodynamic modeling uncertainties. It is likely that $\mathrm{CM}$ angular rates will have to be monitored during the coast phase to provide for an early chute deploy should the motion become excessive. Despite these difficulties, the test will be successful even if aerodynamic damping data are gathered only about a small alpha range around trim, as it will be the only flight data of this type that have been gathered. If data for fewer than three oscillations are collected, the system identification can still be done, but the model uncertainty will increase.

\section{Monte Carlo Analysis}

To establish the overall feasibility of the proposed MLAS ascent abort flight test and to assess FTV performance, a Monte Carlo analysis was performed using the 6-degree-of-freedom POST2 simulation. The simulation was initiated at escape-motor ignition and included the entire FTV escape trajectory and subsequent CM free flight up until deployment of the drogue parachutes. This simulation included a comprehensive aerodynamic database that modeled the static force and moments of the FTV at subsonic, transonic, and supersonic flight conditions over a full 0- to 180-degree alpha range. The CM free-flight segment was modeled using the Orion aerodynamic database, which included both static and dynamic aerodynamic coefficients. ${ }^{10}$ In addition, the simulation modeled the variation of mass, c.g. and inertia terms, and the varying effects of atmosphere and winds using the Global Reference Atmosphere Model (GRAM 2010). ${ }^{11}$

A basic feedback control system was integrated into the simulation for stability and control. The controller followed open-loop attitude guidance commands to perform initial steering during the escape motor burn and was also used during ACS thruster operation to maintain control during coasting segments, perform the reorientation maneuver, and hold the FTV at the required trim offset attitude prior to fairing jettison. The simulation included sufficient fidelity to ensure that the TVC deflection and rate limits were not violated and that the minimum on/off times of the ACS thrusters were maintained.

For this study, it was assumed that the only sensors available were from the INS and that there was no way to measure air data directly. Consequently, the controller did not account for winds when targeting alpha and angle-ofsideslip (beta) guidance commands immediately before and after the reorientation maneuver. This limitation was deemed acceptable for this initial feasibility study and alternatives such as adding sensors to measure air data and modifying the control algorithm to estimate alpha/beta may be explored in future studies. The addition of an air data 
system would also benefit the CM free-flight test by decreasing the uncertainty of estimated aerodynamic models derived from system identification.

The input dispersions and uncertainties assumed in the flight-test Monte Carlo analysis are listed in Table 2. Of particular importance was accurate modeling of the dispersions in propellant burn rate for the solid rocket escape motors. Small variations in the burn rate between the six different escape motors had the potential to induce significant thrust moments throughout the burn but especially at burnout. By modeling this effect in the simulation, requirements for custom thrust traces could be developed that minimized the induced thrust moments for given burn rate dispersions, and the control system could be made robust enough to accommodate the disturbances. Another important source of uncertainty was in the flight conditions at the time of abort initiation. These uncertainties arise from dispersions in the boost trajectory. Since booster Monte Carlo analyses were not performed in this study, these uncertainties were estimated.

Table 2. Monte Carlo Uncertainties.

\begin{tabular}{|c|c|c|c|c|}
\hline Dispersion & Units & Mean & $\begin{array}{c}\text { +/- Range } \\
\text { or } \\
\text { +/- 3-sigma } \\
\end{array}$ & Distribution \\
\hline Ignition Delay for each Escape Motor & sec & 0.0045 & 0.002 & normal \\
\hline Propellant Mean Bulk Temperature for each Escape Motor & deg & 70 & 1 & normal \\
\hline Propellant Burn Rate scale factor for each Escape Motor & - & 1.00 & 0.05 & normal \\
\hline FTV Aero Coefficents Multipliers (CA \& CN) & - & 1.00 & 0.10 & uniform \\
\hline FTV Aero, Xcp shift & in & 0.0 & 6.0 & uniform \\
\hline CM Aero Coefficients & \multicolumn{4}{|c|}{ Dispersed using MPCV Aerodynamic Database } \\
\hline Xcg shift & in & 0.0 & 1.1 & normal \\
\hline Ycg, Zcg shift & in & 0.0 & 0.5 & normal \\
\hline Vehicle weight scale factor & - & 1.000 & 0.018 & uniform \\
\hline Ixx scale factor & - & 1.000 & 0.042 & normal \\
\hline Iyy scale factor & - & 1.000 & 0.064 & normal \\
\hline Izz scale factor & - & 1.000 & 0.066 & normal \\
\hline Ixy bias & slug- $\mathrm{ft} 2$ & 0 & 296 & uniform \\
\hline Ixz bias & slug- $\mathrm{ft} 2$ & 0 & 298 & uniform \\
\hline lyz bias & slug- $\mathrm{ft} 2$ & 0 & 265 & uniform \\
\hline Abort initiation altitude & $\mathrm{ft}$ & 40,516 & 2600 & normal \\
\hline Abort initiation velocity & $\mathrm{ft} / \mathrm{s}$ & 1,584 & 85 & normal \\
\hline Abort initiation flight path angle & deg & 27.777 & 1.000 & normal \\
\hline Abort initiation alpha total & deg & 0.0 & 2.0 & normal \\
\hline Abort initiation aerodynamic roll angle & deg & 0.0 & 180.0 & uniform \\
\hline Abort initiation pitch,yaw,roll rates & $\mathrm{deg} / \mathrm{s}$ & 0.0 & 3.0 & normal \\
\hline Atmosphere/Wind & \multicolumn{4}{|c|}{ Dispersed using Gram 2010} \\
\hline
\end{tabular}

A 1000-case Monte Carlo analysis was performed using the randomized inputs described in Table 2. The purpose of this analysis was to demonstrate an initial level of feasibility of the FTV concept and the flight-test sequence as a whole. It is expected that additional refinements to the design and further analysis will be required as the concept matures. Successful cases exhibited stable flight throughout the test trajectory with no excessive oscillations or tumbling during the initial pitch maneuver or reorientation. Also, a Q-Alpha-Total structural load indicator was required to remain below 10,000 psf-deg from abort initiation to fairing jettison. In addition, TVC deflections were required to remain below 14.5 degrees and ACS propellant usage had to be less than $95 \%$ of the total reserve. Finally, for the duration of the $\mathrm{CM}$ free flight-test segment, the total alpha had to remain greater than 130 degrees (i.e., within \pm 50 degrees of a heat shield forward orientation) to ensure that drogue deploy limits were satisfied. For this feasibility study, it was required that $95 \%$ of Monte Carlo cases satisfy all of these constraints.

Monte Carlo results for these various metrics are shown in Table 3 . The $95 \%$ success target was met for all metrics. Of the 1000 random cases, 14 tumbled during the initial pitch maneuver prior to reorientation. These loss-of-control cases occurred primarily because of unexpected thrust moments caused by large thrust dispersions due to variations in burn rate between the six different escape motors. The 14 cases were not included in the evaluation of the remaining metrics reported in Table 3. There were no cases that violated the load metric or the ACS propellant usage requirements, but seven cases had large TVC deflections that exceeded the deflection limit and are discussed in more detail below. Finally, there were also seven cases that violated the total alpha requirement during the CM free-flight phase when the capsule was oscillating about its trim point. The large amplitude oscillations were likely due to aerodynamic uncertainties and/or a larger than expected trim offset at the start of the free-flight phase (due to a lack of knowledge of winds). These violations can potentially be eliminated with the addition of an air data system or use 
of a method for estimating alpha and beta. It may also be possible to initiate drogue deployment early (when the vehicle is approaching the deployment limits) with the only cost being the loss of some of the aerodynamic damping data.

Table 3. Monte Carlo Results (1000 total cases, 986 non-tumble cases).

\begin{tabular}{|c|c|}
\hline Metric & Result \\
\hline No. of cases that tumble prior to reorientation & 14 \\
\hline No. of non-tumble cases with Q-alpha-total > 10,000 psf-deg & 0 \\
\hline No. of non-tumble with TVC Deflections > 14.5 deg & 7 \\
\hline No. of non-tumble cases exceeding 95\% ACS Propellant & 0 \\
\hline No. of non-tumble cases exceeding 130 deg total alpha during CM free flight & 7 \\
\hline
\end{tabular}

Figure 13 shows time history profiles of altitude, Mach number, dynamic pressure, and total alpha for all 986 of the Monte Carlo cases that did not tumble. The total alpha plot shows that there are several cases that did not tumble but still exhibited large total alpha excursions shortly after the initial pitch maneuver. Some dispersed cases also had high TVC deflections due to large disturbance moments during escape-motor burnout; however, there was enough control authority to keep the cases from tumbling. It is likely that the number of cases that tumble or have large pitch excursions could improve with control system refinements such as the inclusion of an alpha/beta estimator. In addition, further improvements may also be possible with a less aggressive pitch maneuver since the minimum separation distance at 3 seconds is well above the 175 -foot requirement.
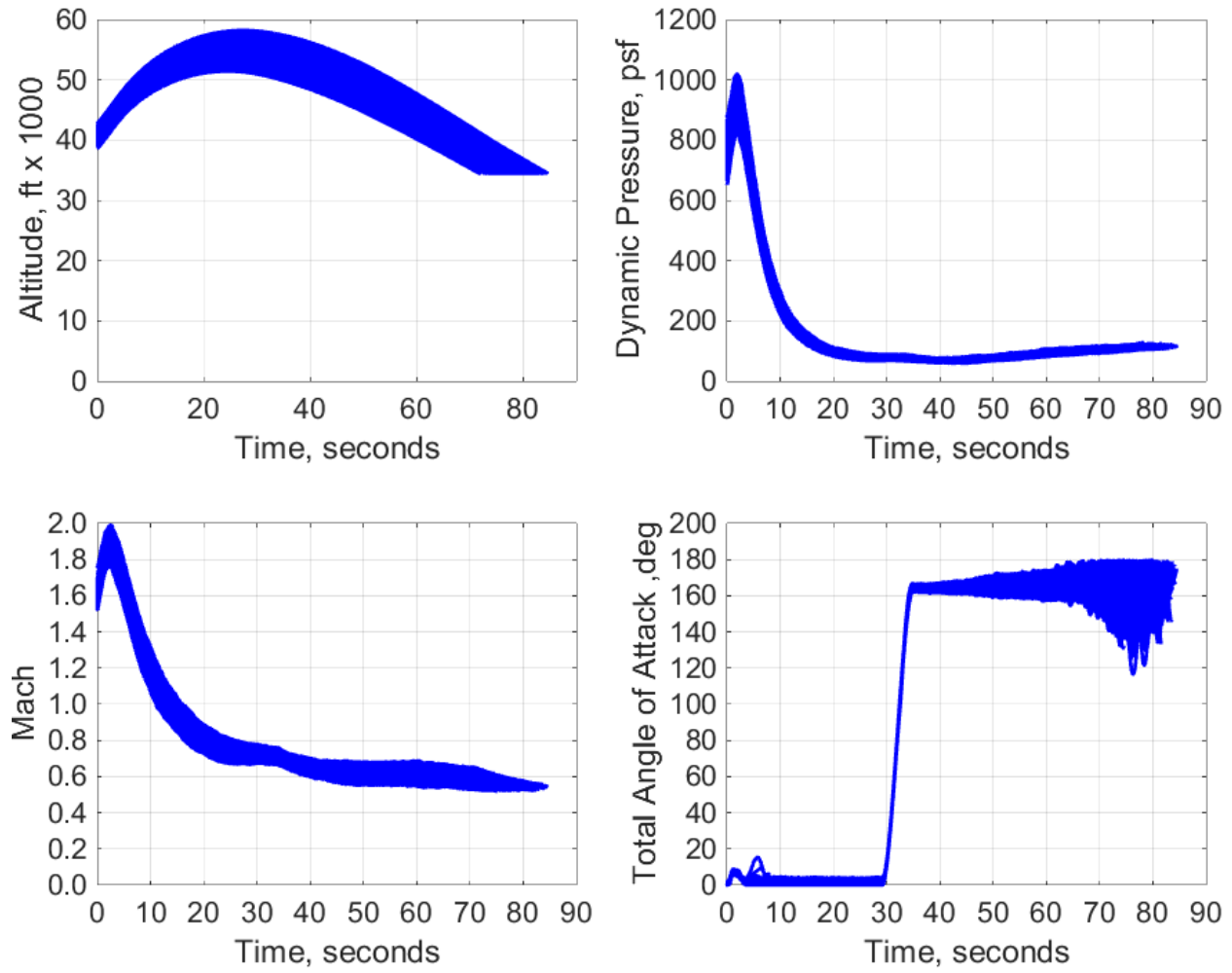

Figure 13. Monte Carlo profiles.

Figure 14 better shows the alpha and beta variation during the CM free-flight phase. The results illustrate the uncertainty in the initial trim offset as there are numerous cases that start on both sides of the trim point. Still, most cases exhibit the desired oscillatory behavior and complete three full oscillation cycles while remaining above the 130-degree total alpha limit (as seen in Fig. 13). 

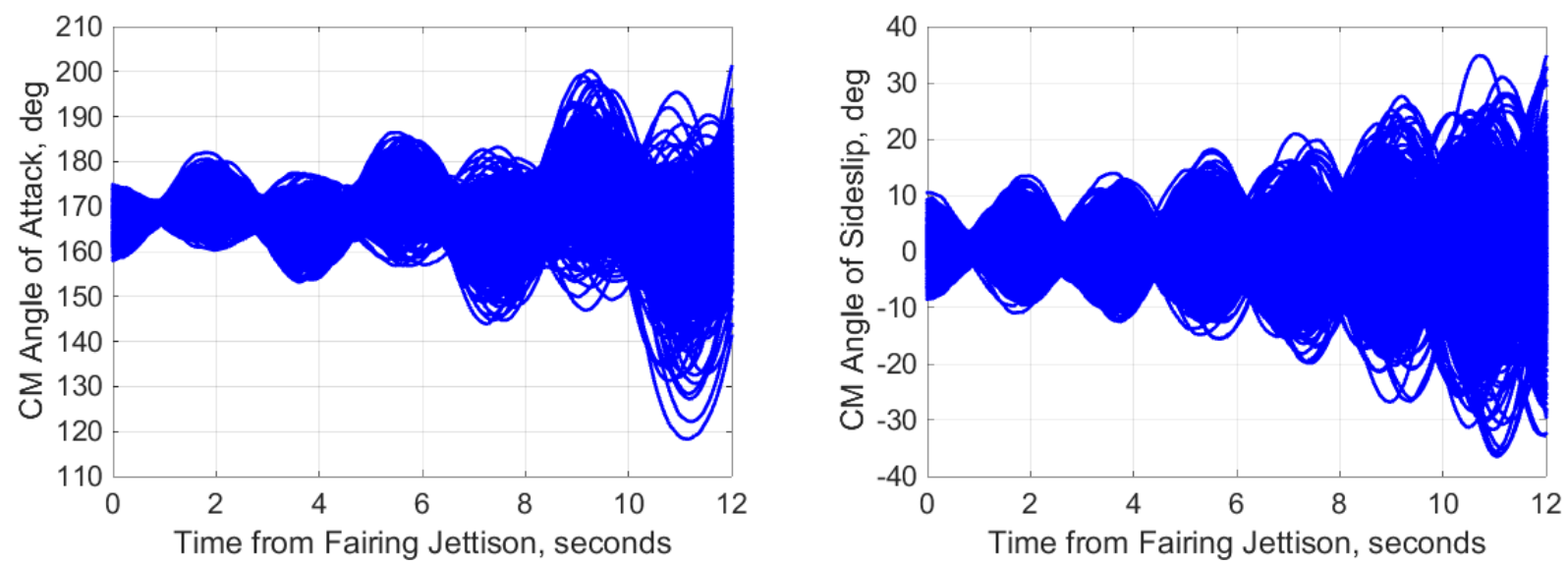

Figure 14. Monte Carlo profiles of $\mathrm{CM}$ alpha and beta during free-flight phase for the $\mathbf{9 8 6}$ non-tumble cases.

Histograms of the peak Q-Alpha-Total and ACS propellant usage are shown in Fig. 15 for the non-tumble cases. Q-Alpha-Total was a load indicator and was required to remain below 10,000 psf-deg to ensure that structural load limits were not violated. The highest loads typically occurred before reorientation, during the pitch maneuver. The histogram shows that there were no violations of the load indicator and the highest cases still had $\sim 2,000$ psf-deg of margin. Similarly, the ACS propellant usage histogram indicates that most cases used less than half the available ACS propellant, suggesting it may be possible to reduce the amount of ACS propellant.
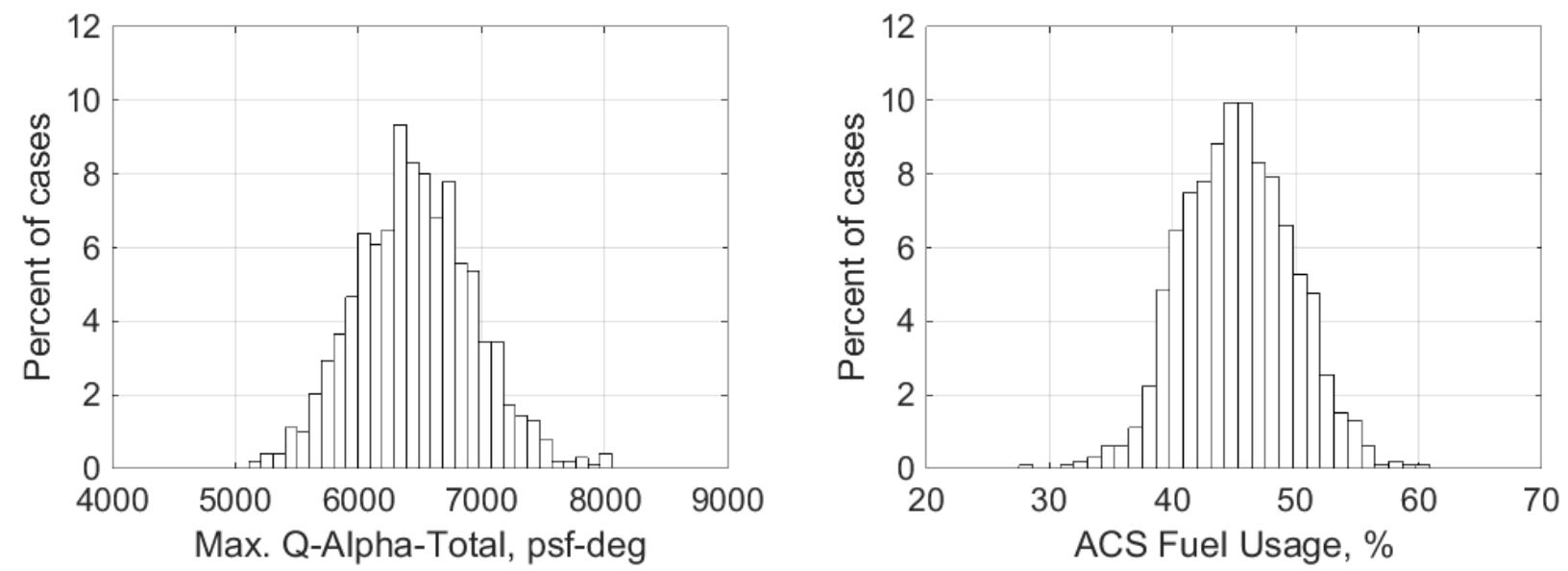

Figure 15. Histograms of maximum Q-Alpha-Total and percent of ACS fuel remaining at escape fairing jettison for the 986 non-tumble cases.

\section{Conclusion}

Crew safety is a key goal as NASA seeks to conduct and promote the development of new human-rated space transportation options. Future launch systems must provide a robust crew escape capability from the pad to orbit insertion while limiting the impact on overall system performance. To address this challenge, the NASA Engineering and Safety Center conducted the Max Launch Abort System (MLAS) Project to explore alternate crew escape vehicle concepts that eliminate the conventional escape tower derived from heritage systems. Eliminating the escape tower has the potential to greatly improve the overall integration of the escape system with the launch vehicle, potentially lowering structural weights, reducing bending moments, and decreasing aero-acoustic loads.

The MLAS escape vehicle is an all-propulsive, actively controlled towerless crew escape system built into an aerodynamic/structural fairing that fully encapsulates the crew module. An ascent abort test has been proposed to demonstrate escape capability at a realistic, high dynamic pressure flight condition. Although costly, it is recognized that meeting NASA's stringent safety requirements will likely require a full-scale test to demonstrate end-to-end performance.

The flight test was designed to maximize its value and data return, and primary science objectives were defined that addressed specific risk areas in the vehicle design. Of particular interest was characterizing potential aeropropulsive jet interactions due to the pulsing operation of the liquid attitude control thrusters. The selection of the 
booster and design of its trajectory were instrumental in ensuring that jet interaction data would be collected across the transonic regime, where performance uncertainties were highest, and not just at a single flight condition. To further enhance the overall value of the flight test, secondary objectives were added to obtain aerodynamic damping data for the crew module and perform a high-altitude parachute deployment. These additional objectives, which occurred after escape and did not involve the MLAS vehicle, required careful adjustment of the escape trajectory to ensure they could be met without negatively affecting the primary goal of validating escape functionality. Monte Carlo analysis was performed to establish the feasibility of the overall test and provide confidence that test objectives could be met.

\section{Acknowledgments}

The authors would like to thank Bruce Owens and Eugene Morelli for providing their expertise and support in the development of the crew module free flight, Dan Yuchnovicz for consultation on the parachute descent and Dee Bullock for preparation of the final manuscript.

\section{References}

${ }^{1}$ Townsend, N. A., “Apollo Experience Report - Launch Escape Propulsion Subsystem,” NASA Technical Note D-7083, Washington D.C., March 1973.

${ }^{2}$ McCarthy, J. F., Dodds, J. I., and Crowder, R. S., "Development of the Apollo Launch Escape System,” Journal of Spacecraft, Vol. 5, No. 8, pp. 927-932.

${ }^{3}$ Sullivan, R. B., Bocam, K., and Escalera, P., "Development of the Orion PA-1 Launch Abort System - From Conception to Demonstration," AIAA 2011-7129.

${ }^{4}$ Dennehy, C. J., Lanzi, R. J., and Ward, P. R., “GN\&C Design Overview and Flight Test Results from NASA's Max Launch Abort System (MLAS)," AIAA 2010-9432.

${ }^{5}$ Tartabini, P. V., Gilbert, M. G., and Beaty, J. R., "Flight Performance Feasibility Studies for the Max Launch Abort System," AIAA 2013-4685.

${ }^{6}$ Stivers, L. S. and Spencer, B., "Studies of Optimum Body Shapes at Hypersonic Speads,” NASA TN-D-4191, Oct. 1967.

${ }^{7}$ Brauer, G. L., Cornick, D. E., and Stevenson, R., "Capabilities and Applications of the Program to Optimize Simulated Trajectories (POST),” NASA CR-2770, February 1977.

${ }^{8}$ Owens, D. B. and Aubuchon, V. V., "Overview of Orion Crew Module and Launch Abort Vehicle Dynamic Stability," AIAA 2011-3504.

${ }^{9}$ Klein, V. and Morelli, E. A., Aircraft System Identification - Theory and Practice, AIAA Education Series, AIAA, Reston, VA, 2006.

${ }^{10}$ Bibb, K. L., Walker, E. L., Brauckmann, G. J., and Robinson, P. E., "Development of the Orion Crew Module Static Aerodynamic Database, Part II: Supersonic/Subsonic,” AIAA 2011-3507.

${ }^{11}$ Leslie, F. W., and Justus, C. G., "The NASA Marshall Space Flight Center Earth Global Reference Atmospheric Model 2010 Version," NASA TM 2011-216467, June 2011.

${ }^{12}$ Dennehy, N. C., Yuchnovicz, D. E., Lanzi, R. J., Ward, P. R., and Shreves, C. M., "Design Experiences and Flight Test Results from NASA's Max Launch Abort System (MLAS): A Flight Mechanics Perspective,” AAS 10-084. 